\title{
The response regulator PhoP4 is required for late developmental events in Myxococcus xanthus
}

\author{
Correspondence \\ Mitchell Singer \\ mhsinger@ucdavis.edu
}

Received 29 December 2005

Revised 23 February 2006

Accepted 26 February 2006

\author{
Vinh D. Pham, ${ }^{1} \dagger$ Conrad W. Shebelut, ${ }^{1} \ddagger$ Ivy R. Jose, ${ }^{1}$ David A. Hodgson, ${ }^{2}$ \\ David E. Whitworth ${ }^{2}$ and Mitchell Singer ${ }^{1}$ \\ ${ }^{1}$ Section of Microbiology and Center for Genetics and Development, 268 Briggs Hall, \\ University of California, Davis, CA 95616, USA \\ ${ }^{2}$ Department of Biological Sciences, University of Warwick, Coventry CV4 7AL, UK
}

\begin{abstract}
Phosphate regulation is complex in the developmental prokaryote Myxococcus xanthus, and requires at least four two-component systems (TCSs). Here, the identification and characterization of a member of one TCS, designated PhoP4, is reported. phoP4 insertion and in-frame deletion strains caused spore viability to be decreased by nearly two orders of magnitude, and reduced all three development-specific phosphatase activities by 80-90\% under phosphate-limiting conditions. Microarray and quantitative PCR analyses demonstrated that PhoP4 is also required for appropriate expression of the predicted pstSCAB-phoU operon of inorganic phosphate assimilation genes. Unlike the case for the other three M. xanthus Pho TCSs, the chromosomal region around phoP4 does not contain a partner histidine kinase gene. Yeast two-hybrid analyses reveal that PhoP4 interacts reciprocally with PhoR2, the histidine kinase of the Pho2 TCS; however, the existence of certain phenotypic differences between phoP4 and phoR2 mutants suggests that PhoP4 interacts with another, as-yet unidentified, histidine kinase.
\end{abstract}

\section{INTRODUCTION}

The social bacterium Myxococcus xanthus undergoes a multicellular morphological differentiation process when starved of nutrients. This process requires precise spatial and temporal regulation for the cells to elaborate macroscopic structures, called fruiting bodies, within which vegetative cells differentiate into stress-resistant cysts called myxospores. For an M. xanthus swarm to determine the necessity for, and appropriateness of, activating this developmental programme, multiple nutritional and population-density signals must be monitored (for reviews, see Kaiser, 2000; Shimkets, 1999; Ward \& Zusman, 2000).

One way in which bacteria sense and process such environmental signals is through signal transduction pathways known as two-component systems (TCSs). These typically consist of a sensor histidine kinase and a partner effector protein called the 'response regulator'. For most TCSs, the pathway becomes activated upon detection of a signal by the histidine kinase, which then autophosphorylates a conserved histidine residue in its C-terminal H-box domain.

tPresent address: Department of Civil and Environmental Engineering, Massachusetts Institute of Technology, Cambridge, MA 02139, USA.

$\ddagger$ Present address: Department of Molecular Biology, Princeton University, Princeton, NJ 08544, USA.

Abbreviations: $\mathrm{Ml}$, magnesium-independent; $\mathrm{QPCR}$, quantitative PCR; TCS, two-component system.
The signal is subsequently transduced by transfer of the phosphoryl group from the H-box histidine to a conserved aspartate residue in the receiver domain of the partner response regulator. In its phosphorylated form, the response regulator effects a variety of physiologic changes in the cell, which usually involve the activation or repression of genes to coordinate an appropriate response to the activating signal (for reviews, see Kenney, 2002; Parkinson \& Kofoid, 1992; Stock et al., 2000).

The recently completed genome of $M$. xanthus reveals the presence of 255 known and putative TCS components, $44 \%$ of which are orphaned or unpartnered (D. E. Whitworth, unpublished data). One of these is a putative response regulator that we have named $\mathrm{PhoP} 4$, for phosphate regulation protein 4. It shows homology to PhoB in Escherichia coli and PhoP in Bacillus subtilis, which in those organisms regulate the Pho regulon of phosphate-starvation-inducible genes, including genes for phosphate transport and several phosphatases (Hulett et al., 1994; Makino et al., 1989).

The regulation of phosphate pools is important for development in $M$. xanthus, as evidenced by the fact that starvation for inorganic phosphate induces fruiting-body formation (Manoil \& Kaiser, 1980). Furthermore, phosphatase activities with $\mathrm{pH}$ optima at $5 \cdot 2,7 \cdot 2$ and $8 \cdot 5$, known as the magnesium-independent (MI) acid, MI neutral and MI alkaline activities, respectively, have been reported that are specifically activated in development at discrete times 
following fruiting-body formation (Weinberg \& Zusman, 1990). The tight temporal regulation of these MI phosphatase activities suggests that they play an integral role in development; however, the number and identities of all the phosphatases contributing to these activity profiles remain to be elucidated.

Previously, the laboratory of Muñoz-Dorado identified and characterized three known Pho TCS operons named phoP1phoR1, phoR2-phoP2 and phoR3-phoP3 (Carrero-Lerida et al., 2005; Martinez-Canamero et al., 2003; MoraledaMuñoz et al., 2003). Deletions in any of these systems affect specific developmental phosphatase activity patterns in $M$. xanthus, while leaving the vegetative phosphatase activities, which are magnesium dependent, unchanged. In addition, none of these mutations appears to affect sporulation.

We report here the identification and characterization of a member of a fourth Pho TCS, PhoP4. Our data show that PhoP4 is required for (I) expression of all three known development-specific phosphatase activities, (II) wild-typelevel sporulation, and (III) expression of the predicted assimilatory inorganic phosphate pstSCAB-phoU operon. In addition, our yeast two-hybrid analyses show reciprocal interactions between PhoP4 and the histidine kinase of another M. xanthus Pho TCS, PhoR2. In sum, these data indicate that PhoP4 (and the TCS system it represents) is a very important component of the M. xanthus Pho regulatory circuit, specifically with regard to activities required for development.

\section{METHODS}

Strains, plasmids, cloning, and transformation. A complete list of strains and plasmids used in this study is shown in Table 1. Plasmids were propagated in E. coli JM109 (Yanisch-Perron et al., 1985). An internal fragment of the phoP4 ORF was amplified by PCR using forward primer PhoP4-F43 and reverse primer PhoP4R575, which contain engineered PstI and EcoRI restriction sites (underlined, see Table 2), respectively. The resultant amplicon was digested with the two restriction enzymes and inserted directionally into vector pBGS18 to generate plasmid pDV525. Wild-type $M$. xanthus strain DK1622, which does not maintain E. coli vectors, was transformed by electroporation with pDV525 using a GenePulser Xcell electroporator (Bio-Rad Laboratories), as described elsewhere (Kashefi \& Hartzell, 1995). Kanamycin-resistant $\left(\mathrm{Km}^{\mathrm{R}}\right)$ transformants were selected and then screened by Southern blot analysis for the insertion of pDV525 by homologous recombination to generate the phoP4:: pDV525 insertion strain VP965.

The phoP4 in-frame deletion strain was generated by allelic exchange using the GalK selection method (Ueki et al., 1996). Briefly, sequences upstream and downstream of the phoP4 gene were amplified by PCR using the primers listed in Table 2, then ligated into the multiplecloning site of plasmid pBJ114 to form plasmid pCS66. Insertion of this plasmid at the phoP4 locus was accomplished as described above. $\mathrm{Km}^{\mathrm{R}}$ transformants were plated on $2 \%$ galactose CTTYE agar (CTT supplemented with $0 \cdot 2 \%$ yeast extract) and $\mathrm{Km}^{\mathrm{S}} \mathrm{Gal}^{\mathrm{R}}$ candidates (which had lost the plasmid by homologous recombination) were screened by Southern blot analysis for the presence of the deletion allele, yielding strain VP963, in which phoP4 was deleted between bases 77 and 541.
Plasmids pDV888, pDV890 and pDV892 were generated by cloning the PstSqF1/R1, PstBqF1/R1 and PhoUqF1/R1 PCR amplicons, respectively, into the pCR 2.1-TOPO vector as directed by the TOPO TA Cloning Kit protocol (Invitrogen).

Culture media and conditions. E. coli strains were grown in Luria-Bertani broth (Sambrook et al., 1989) in a roller drum at $37^{\circ} \mathrm{C}$ and 60 r.p.m. Unless otherwise stated, M. xanthus strains were grown on CTTYE media at $33^{\circ} \mathrm{C}$, as previously described (Hodgkin $\&$ Kaiser, 1977). When needed, $40 \mu \mathrm{g}$ kanamycin monosulfate (Sigma-Aldrich) was added per millilitre of medium.

Developmental media and conditions. Mid-exponential-phase M. xanthus cells were concentrated to a density of $5 \times 10^{9} \mathrm{cells}^{-1}$, and spotted onto TPM agar $(10 \mathrm{mM}$ Tris, $\mathrm{pH} 7 \cdot 6,1 \mathrm{mM}$ potassium phosphate, pH 7·8, $8 \mathrm{mM}$ magnesium sulfate; Kroos et al., 1986) at $33^{\circ} \mathrm{C}$ for developmental and sporulation assays. Fruiting-body formation was visually evaluated by light microscopy with a Nikon SMZ800 microscope. Myxospore preparations were performed as previously described (Pham et al., 2005).

Yeast two-hybrid assays. The Saccharomyces cerevisiae strain used was EGY48 (Gyuris et al., 1993). Cultivation and yeast two-hybrid methods were as described previously (Whitworth \& Hodgson, 2001). Briefly, DNA encoding the receiver domains and $\mathrm{H}$ boxes of pho genes was amplified by PCR according to a protocol described by Whitworth \& Hodgson (2001), using the primers listed in Table 2. PCR products were cloned into both pEG202 and pJG4-5, generating LexA and activation domain (AD) fusions, respectively (Table 1), using EcoRI and XhoI restriction sites. Testing the suitability of all LexA fusions for use in the two-hybrid system and subsequent screening for protein-protein interactions were also as described by Whitworth \& Hodgson (2001). A positive control was provided by plasmids pDEW002 and pDEW020 (Table 1), encoding a pair of fusion proteins known to interact strongly and specifically (Browning et al., 2003). Due to the semi-quantitative nature of the yeast two-hybrid assay, interactions were assessed visually on medium containing $\mathrm{X}-\mathrm{Gal}$, with the degree of indigo colouration scored in the range 1-3, as previously reported (Browning et al., 2003; Higgs et al., 2005; Whitworth \& Hodgson, 2001). When tested with ONPG as the substrate, typical ' 1 ', ' 2 ', and ' 3 ' interactions were found to express $\beta$-galactosidase with specific activities of $2 \cdot 9 \pm 0 \cdot 6$, $10 \cdot 7 \pm 1 \cdot 0$ and $2862 \pm 1962 \mathrm{nmol}(\mathrm{mg} \text { protein })^{-1} \mathrm{~min}^{-1}$, respectively. The lowest specific activities, at $0 \cdot 7 \pm 0 \cdot 3$, corresponded to the absence of interaction, which is indicated with a - in Table 4.

RNA isolation. Total RNA samples used for all procedures described in this study were extracted by the hot phenol method (Sambrook et al., 1989) from approximately $10^{10}$ quick-frozen $M$. xanthus cells. These cells were harvested either during vegetative growth at a cell density of $5 \times 10^{8}$ cells $\mathrm{ml}^{-1}$, or 48 and $60 \mathrm{~h}$ after the initiation of starvation on TPM agar plates.

Quantitative PCR (QPCR). Analysis was performed as described previously (Diodati et al., 2006). Expression of the pstS, pstB and phoU genes was calculated from DNA quantities obtained for concurrent standard curve reactions. Typically, a standard curve was generated by reacting a 10 -fold dilution series of plasmid DNA (pDV888 for $p s t S$, pDV890 for $p s t B$, and pDV892 for $p h o U$ ), ranging from $10^{10}$ to $10^{1}$ copies per microlitre, with the appropriate primer set and the DyNAmo HS SYBR Green QPCR Master Mix (Finnzymes).

DNA microarrays. PCR-generated DNA microarrays containing probes to the $7235 \mathrm{M}$. xanthus ORFs of the M1 genome (Diodati et al., 2006; Jakobsen et al., 2004) were spotted onto poly-L-lysine coated glass slides by the Stanford Functional Genomics Facility (Stanford, CA). Samples from three independent biological replicates were collected as described above, and DNA microarray analyses were performed as previously described (Diodati et al., 2006). Hierarchical 
Table 1. Strains and plasmids

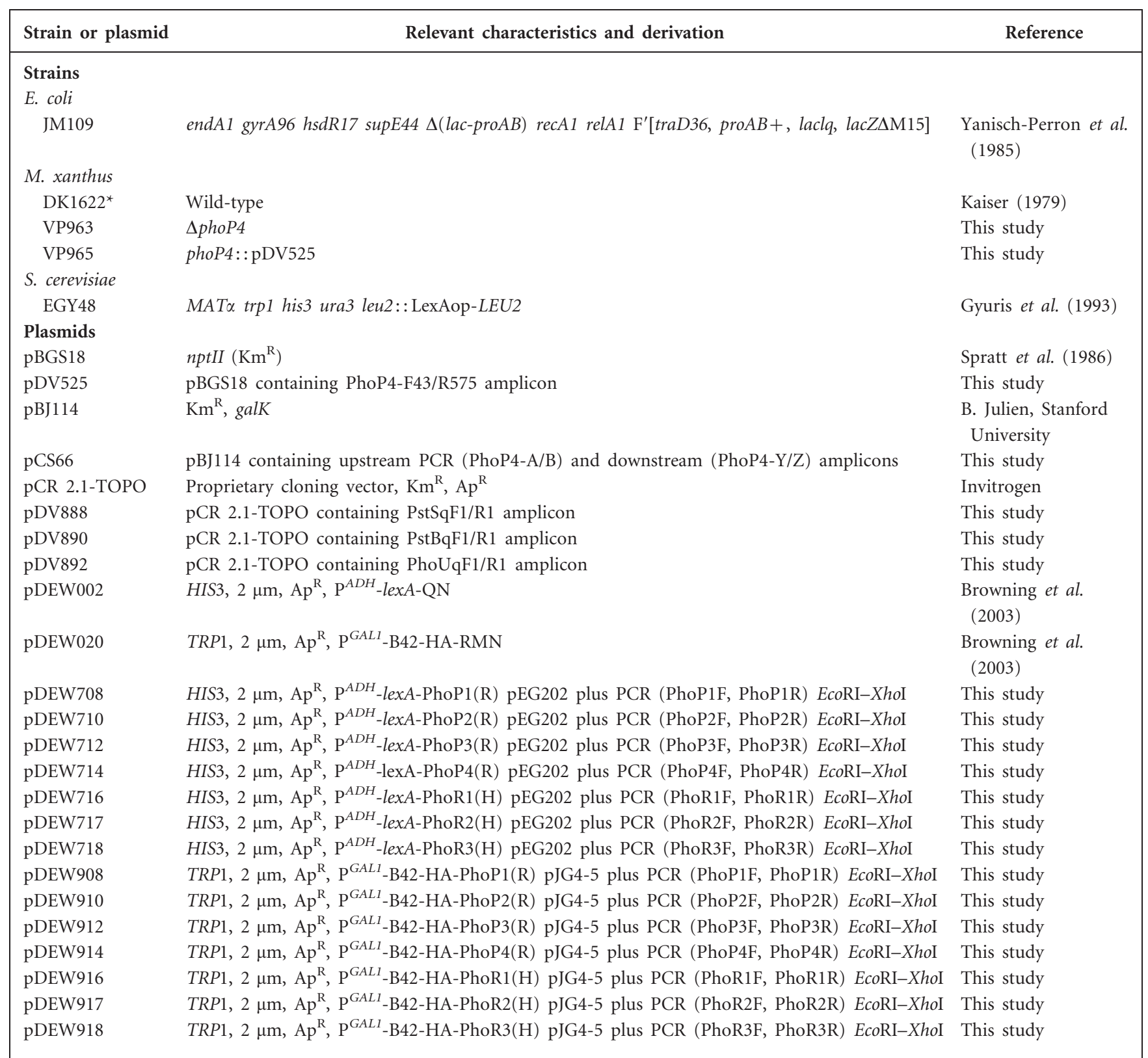

${ }^{\star}$ DK1622 was the parent strain for both phoP4 mutants.

clustering and statistical analyses were performed using Cluster and Java TreeView software (Saldanha, 2004), and SAM (Tusher et al., 2001). All DNA microarray data have been submitted to the Gene Expression Omnibus (GEO) at the National Center for Biotechnology Information (NCBI) (http://www.ncbi.nlm.nih.gov/projects/ geo/), and accession numbers are provided in the legend of Fig. 3.

Phosphatase assays. Studies of other bacterial model organisms have shown that $\mathrm{PhoB} / \mathrm{PhoP}$ regulation of phosphatases is most significant under phosphate-limiting conditions (Antelmann et al., 2000; Hirani et al., 2001; Wanner, 1996). Phosphatase assays were performed as previously described (Weinberg \& Zusman, 1990), with the exception that cells used for the assay were cultured in CTT media without additional phosphate. We previously reported that CTT medium without added phosphate contains $\sim 0.75 \mathrm{mM}$ phosphate (all of it presumably derived from casitone), while fully supplemented CTT medium contains 1.75 mM phosphate (Harris et al., 1998). To perform the phosphatase assay, mid-exponential-phase cells grown on low-phosphate CTT media were concentrated to $5 \times 10^{9} \mathrm{cells} \mathrm{ml}^{-1}$, spotted in triplicate sets onto non-phosphate-supplemented CF agar plates (Hagen et al., 1978), and harvested at various time-points into TM buffer (TPM, buffer without the potassium phosphate) or $20 \mathrm{mM}$ HEPES buffer. Cells were disrupted mechanically by sonication (Branson Sonifier 450, Branson Ultrasonics) with glass beads, and cell and spore breakage was confirmed by observation under light microscopy. Aliquots of cell lysates were added to appropriate buffers containing $0.01 \mathrm{M} \mathrm{p}$-nitrophenol phosphate, incubated at $37^{\circ} \mathrm{C}$, and enzymic activity was assessed colorimetrically at $A_{410}$. Protein 
Table 2. Sequences of oligonucleotide primers

Restriction enzyme sites engineered into each primer are underlined. Sites are: GAATTC for EcoRI, AAGCTT for HindIII, CTGCAG for PstI, and CTCGAG for XhoI.

\begin{tabular}{|c|c|c|}
\hline Primer & Sequence $\left(5^{\prime}-3^{\prime}\right)$ & Purpose \\
\hline PhoP4-F43 & GACCTGCAGGAGCTCATTGATTTCAACCTG & phoP4 insertional disruption \\
\hline PhoP4-R575 & ATGGAATTCGTCTCCAGGGAGGATGACAGG & \\
\hline PhoP4-A & GGTAGGAAGCTTGCAGCAGGAGGATGAAGATGA & phoP4 in-frame deletion (upstream amplicon) \\
\hline PhoP4-B & GAGGCCCTGCAGCCGCAGGTTGAAATCAATGAG & \\
\hline PhoP4-Y & AAGAGGCTGCAGGCCTGTCATCCTCCCTGGAGA & phoP4 in-frame deletion (downstream amplicon) \\
\hline PhoP4-Z & ATAGGGGAATTCCGCCTCTTGTGACATTCGTGT & \\
\hline PhoP1F & GAGAGAGAATTCATGTCGCGCATCCTGATTATCG & PhoP1 yeast two-hybrid construct \\
\hline PhoP1R & AAGGAACTCGAGCTAGCGCAGAAGGAGCTCGCGGACG & \\
\hline PhoR1F & GTCGTCGAATTCACCGAGCTGCGCCGGCTGG & PhoR1 yeast two-hybrid construct \\
\hline PhoR1R & CTTCTTCTCGAGTCACAGGGTGACGCGGGCGAGCTT & \\
\hline PhoP2F & CCACCCGAATTCACCGCCACATCCACCCCG & PhoP2 yeast two-hybrid construct \\
\hline PhoP2R & CGTCGTCTCGAGCTAGCGGCGCACCAGGGCGCGTACC & \\
\hline PhoR2F & CCTCCTGAATTCGTGGCCACCGTCTCCCACG & PhoR2 yeast two-hybrid construct \\
\hline PhoR2R & СCTTCCCTCGAGTCAGCGGCCCTTGTCGATGCGG & \\
\hline PhoP3F & GGCGGCGAATTCATGACCTCATCCGTTCCGGCG & PhoP3 yeast two-hybrid construct \\
\hline PhoP3R & TCCTCCCTCGAGTCACGCCAGCAGCTCGCGCAGCCG & \\
\hline PhoR3F & GGCGGCGAATTCCTGGAACTGAAGAGCGAC & PhoR3 yeast two-hybrid construct \\
\hline PhoR3R & CCTTCCCTCGAGTCAGCGCCCCTTGTCGATGCGG & \\
\hline PhoP4F & CACCCAGAATTCGCCATGCCCCATGTCCTCATCATC & PhoP4 yeast two-hybrid construct \\
\hline PhoP4R & ACCACCCTCGAGTCACAGCCGCAGCACCAGCTCCCGC & \\
\hline PstSqF1 & CACCTACGTCTTCGTGAA & pstS QPCR standard curve template \\
\hline PstSqR1 & TGCCGCTCTTGATGTTGT & \\
\hline PstBqF1 & CAGAATCCCCGAGCGAAGATG & pstB QPCR standard curve template \\
\hline PstBqR1 & GCCCGAATGCGTGGTGC & \\
\hline PhoUqF1 & CGACCTGGCGGTGAACA & phoU QPCR standard curve template \\
\hline PhoUqR1 & CGCGAGTCCTCCATCATGTA & \\
\hline
\end{tabular}

concentration was determined by Bradford assay (Bio-Rad Laboratories). For specific activity calculations, extinction coefficients for each buffer were derived empirically.

Sequence analyses. Secondary structure predictions were performed using the GCG suite of sequence analysis tools, version 2 (Accelrys). Amino acid sequences were aligned using the CLUSTALW method (Thompson et al., 1994).

\section{RESULTS}

\section{PhoP4 is a putative PhoB-like response regulator}

PhoP4 (TIGR annotation: MXAN4787) shows 30-40\% sequence identity to PhoB-like response regulators (Fig. 1), which in other bacteria regulate genes needed for phosphate scavenging. PhoP4 is predicted to be 229 amino acids in length, and contains a putative receiver domain between residues 5 and 119 (the putative conserved phosphorylacceptor aspartate is at position 52), and an OmpR-like DNA-binding/transactivation domain between residues 132 and 223. A putative winged helix-turn-helix structure (Okamura et al., 2000), including the recognition helix between residues 194 and 206, is predicted by the method of Chou \& Fasman (1978).

Located 133 bp downstream of the phoP4 gene is the predicted pstSCAB-phoU operon of $\mathrm{Pi}$ assimilatory genes (Fig. 2). Belonging to the $\mathrm{ABC}$ (ATP-binding cassette) superfamily of transporter genes, the pstSCAB-phoU operon encodes a periplasmic binding protein (PstS, MXAN4788), integral membrane-channel proteins (PstC, MXAN4789, and PstA, MXAN4790), an ABC family traffic ATPase (PstB, MXAN4791), and a component hypothesized to participate in $\mathrm{Pi}$ incorporation into ATP (PhoU, MXAN4792). The gene order is identical to that of the homologous E. coli operon; however, in enteric bacteria, the $p h o B$ gene is not located near the pstSCAB-phoU operon (Blattner et al., 1997; Karp etal., 2002).

Additionally, the region upstream of the first gene, pstS, contains at least two candidate PHO-box-like DNA-binding motifs (Fig. 2); these may serve as binding sites for PhoP4, in a similar manner to E. coli $\mathrm{PhoB}$ binding of $\mathrm{PHO}$ boxes in the promoter regions of its target genes, including the pstSCAB-phoU operon (Makino et al., 1996; Wanner, 1996). However, it is important to emphasize that the consensus sequence of a PHO box varies from species to species (Fig. 2; Liu \& Hulett, 1998; Makino et al., 1996; Wanner, 1996), and 

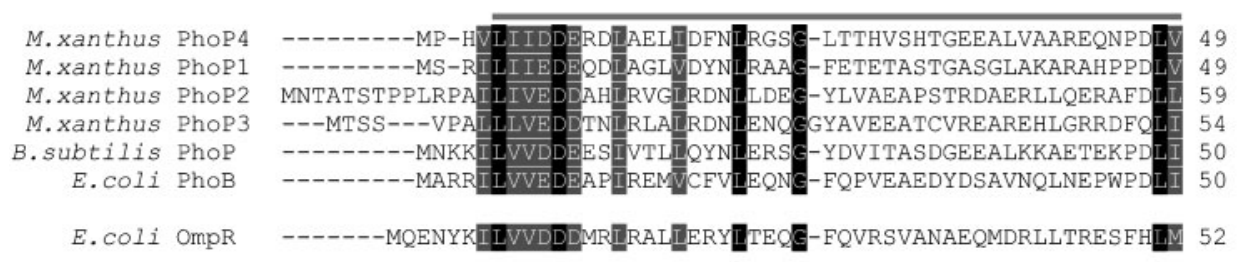

\section{M. xanthus Phop4 M. xanthus PhoP1 M.xanthus PhoP2 M.xanthus PhoP3 B. subtilis PhoP \\ E. COli $\mathrm{PhOB}$ \\ E. coli OmpR}

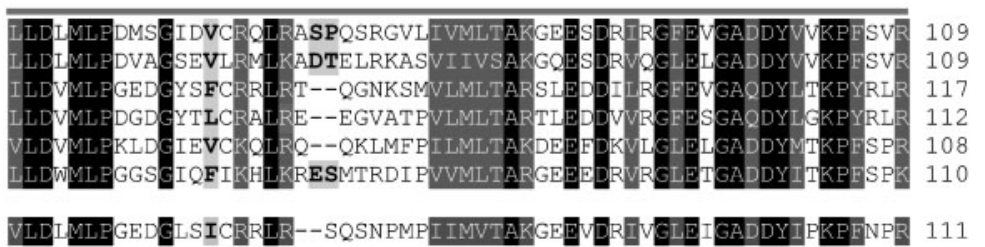

$$
\begin{gathered}
\text { M.xanthus PhoP4 } \\
\text { M.xanthus PhoP1 } \\
\text { M.xanthus PhoP2 } \\
\text { M.xanthus PhoP3 } \\
\text { B.subtilis PhoP } \\
\text { E.coli PhoB } \\
\text { E.coli OmpR }
\end{gathered}
$$

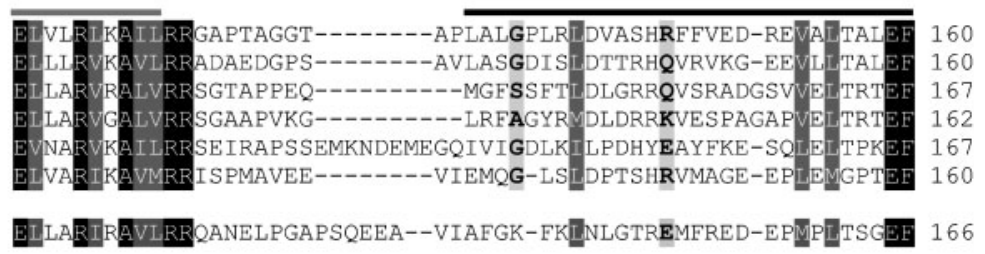
M.xanthus Phop4 M.xanthus Phop1 M.xanthus PhoP2 M. xanthus PhoP3 B. subtilis PhoP E. COli PhoB E. COli OmpR
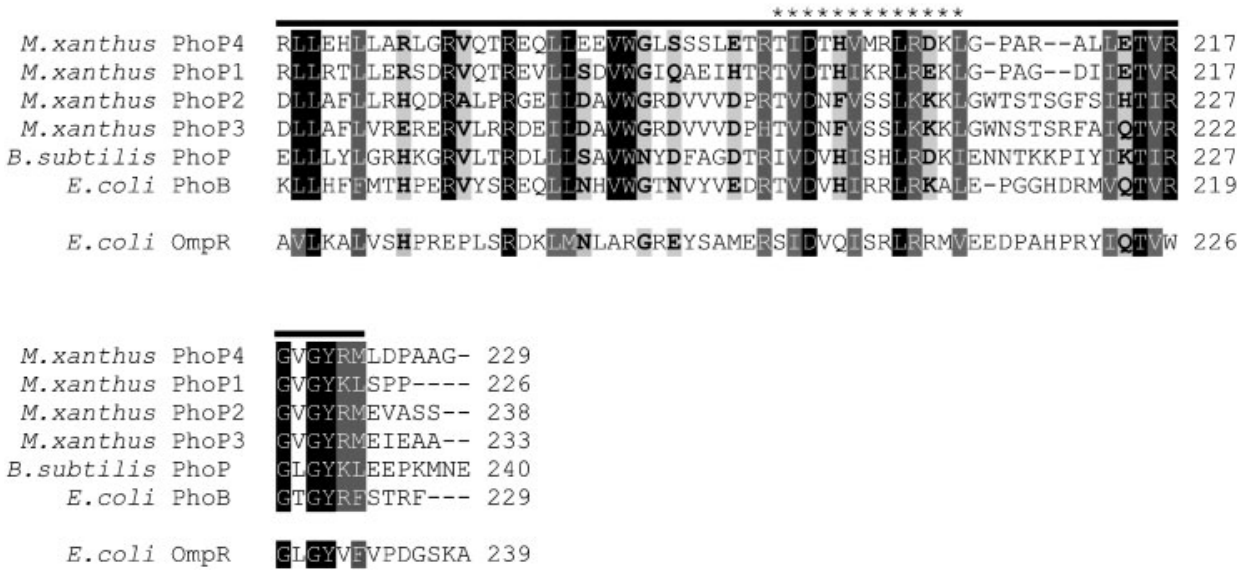

Fig. 1. Amino acid sequence alignment of PhoP4 with other M. xanthus Pho proteins and E. coli PhoB and B. subtilis PhoP. The sequence of the related $E$. coli response regulator $\mathrm{OmpR}$ is shown for comparative purposes. Residues are shaded according to degree of conservation, with black denoting identity, dark grey denoting strong similarity, and light grey denoting 'semiconservation', as defined by the European Bioinfomatics Institute (EBI) (http://www.ebi.ac.uk/clustalw/). Conserved domains are demarcated as grey for the receiver domain, and black for the DNA binding/transactivation domain. The location of the recognition helix is identified by asterisks. Accession numbers are NP417864 for E. coli OmpR, P08402 for E. coli PhoB, P13792 for B. subtilis PhoP, AF133131 for M. xanthus PhoP1, AF157830 for M. xanthus PhoP2 and AF157829 for M. xanthus PhoP3. The PhoP4 sequence is tagged as MXAN4787 on the M. xanthus genome database maintained by The Institute for Genomic Research.

future direct DNA binding studies are needed to evaluate whether any of the candidate sites identified can actually be bound by purified PhoP4 protein.

\section{phoP4 mutants are defective for expression of the predicted pstSCAB-phoU operon}

DNA microarrays were used to compare the patterns of global gene expression between the $\triangle p h o P 4$ mutant and wild-type cells at 0,48 and $60 \mathrm{~h}$ post initiation of development (Fig. 3). During vegetative growth, i.e. at 0 h, no significant differences were observed between wild-type and the $\triangle$ phoP4 mutant cells using DNA microarrays. However, at 48 and $60 \mathrm{~h}$ post initiation, the expression of several genes was affected in the $\Delta p h o P 4$ mutant. The data summarized in Fig. 3 show those genes whose expression either decreased (blue) or increased (yellow) by at least twofold in the $\triangle p h o P 4$ mutant as compared to wild-type. Many of 


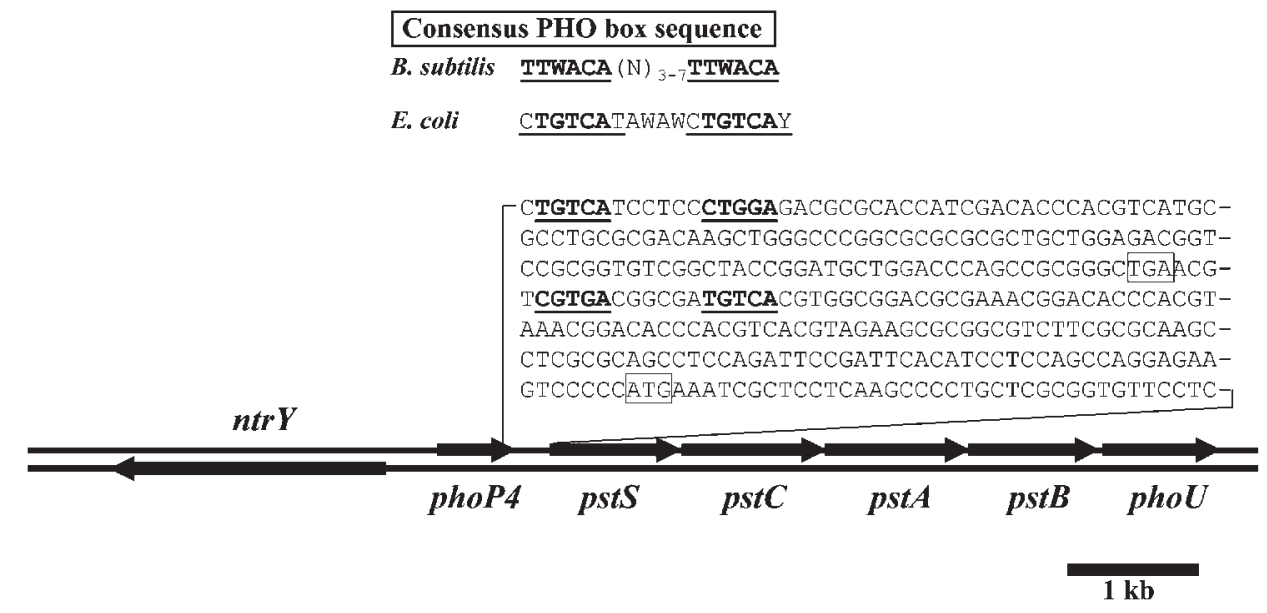

Fig. 2. Map of the phoP4 chromosomal region. ORFs are represented as arrows pointing in the presumed direction of transcription, as defined by The Institute for Genomic Research. The nucleotide sequence of the region between phoP4 and pstS is shown with candidate $\mathrm{PHO}$ boxes composed of dual binding sites (in bold type and underlined); the predicted stop codon for phoP4 (TGA) and the predicted start codon for pstS (ATG) are indicated by boxes. For comparison, the consensus $\mathrm{PHO}$ box sequences in B. subtilis (Liu \& Hulett, 1998) and E. coli (Makino et al., 1996; Wanner, 1996) are also shown. The bases that comprise the binding sites are in bold type and underlined; for $E$. coli $\mathrm{PHO}$ boxes, some authors recognize only the internal heptamer (bold and underlined; Makino et al., 1996), while others define them as encompassing the adjacent bases on each side as well (underlined only; Wanner, 1996). International Union of Biochemistry (IUB) codes for degenerate bases: $\mathrm{W}=\mathrm{A}$ or $\mathrm{T} ; \mathrm{Y}=\mathrm{C}$ or $\mathrm{T} ; \mathrm{N}=\mathrm{A}, \mathrm{C}, \mathrm{G}$ or $\mathrm{T}$.

the genes identified are 'hypothetical proteins'. However, several are genes whose expression is known or predicted to be developmentally regulated, e.g. myxobacterial haemagglutinin (Romeo et al., 1986) and polyketide synthase. Most interesting was the identification of pstS, the putative M. xanthus homologue of the E. coli inorganic phosphate transporter gene. Further analysis revealed that transcription of other genes in the predicted pstSCABphoU operon was reduced by roughly $50 \%$ (Fig. $4 \mathrm{a}$ ). These data were then confirmed and quantified by QPCR using probes to the pstB gene (Fig. $4 \mathrm{~b}$ ): whereas expression of $p s t B$ in the wild-type was more than three times higher at 48 and $60 \mathrm{~h}$ into development than during vegetative growth (at $0 \mathrm{~h}$ ), in the $\triangle p h o P 4$ mutant, the late development $p s t B$ transcript levels remained essentially unchanged compared to the $0 \mathrm{~h}$ time-point. This pattern was very similar to those observed for QPCR analyses of pstS and phoU, the two other genes in the predicted pstSCAB-phoU operon that were examined as part of this study (data not shown).

It should be noted, however, that the immediate downstream location of the predicted pstSCAB-phoU operon raises the possibility that unknown, phoP4-internal cis regulatory elements of the operon could have been removed, disrupted or displaced by the phoP4 mutations (although the candidate PHO boxes identified in Fig. 2 were not affected in either the insertion or in-frame deletion strains). Hence, it is also possible that the expression defects observed by microarray and QPCR described above could have been caused by the inadvertent mutation of unknown cis elements when the phoP4 ORF was knocked out.

\section{PhoP4 is required for development-specific phosphatase activities under phosphate-limiting conditions}

Mutations in the other $M$. xanthus Pho TCSs partially decrease the MI acid phosphatase activity (AcPA) and/or the MI neutral phosphatase activity (NPA), without impairing the MI alkaline phosphatase activity (AlPA) (Carrero-Lerida et al., 2005; Moraleda-Muñoz et al., 2003). In order to determine whether PhoP4 is similarly involved in their regulation, phosphatase activites over developmental timecourses were assayed for both the $\Delta$ phoP4 mutant VP963 and the phoP4::pDV525 insertional disruption mutant VP965, along with the parent wild-type strain DK1622. These analyses revealed that both phoP4 mutants were nearly identically defective for all three developmentspecific phosphatase activites: AcPA and AlPA were down about fivefold compared to wild-type, while NPA was decreased by 10 -fold (Fig. 5). Close examination of the activity profiles also indicated that differences between the wild-type and either phoP4 mutant began to become pronounced at around $44 \mathrm{~h}$ into development, which coincides with the formation of the first myxospores (Fig. 5). This is significant, because it has previously been demonstrated that most development-specific phosphatase activities originate within spores (Weinberg \& Zusman, 1990). In contrast to their effects on the developmental phosphatase 


\begin{tabular}{|c|c|c|c|c|c|c|c|c|c|c|}
\hline \multirow{2}{*}{$\begin{array}{r}\text { Mx number } \\
\text { Down }\end{array}$} & \multirow{2}{*}{$\begin{array}{l}\text { MXAN number } \\
\text { gulated }\end{array}$} & \multicolumn{2}{|c|}{ Time (h) } & \multicolumn{3}{|c|}{$48 \mathrm{~h}$} & \multicolumn{3}{|c|}{$60 \mathrm{~h}$} & \multirow[t]{2}{*}{ Annotation } \\
\hline & & 0 & 60 & Av. & SD & Fold & Av. & SD & Fold & \\
\hline Mx_4548 & MXAN7061 & & & 0.25 & 0.12 & 4.07 & 0.23 & 0.21 & 4.39 & Myxobacterial hemagglutinin \\
\hline Mx_1238 & MXAN4915 & & & 0.25 & 0.10 & 3.93 & 0.49 & 0.45 & 2.03 & Cellulase \\
\hline Mx_1237 & MXAN4915 & & & 0.30 & 0.09 & 3.36 & 0.47 & 0.40 & 2.14 & Laminin A \\
\hline Mx 6464 & MXAN5265 & & & 0.30 & 0.10 & 3.35 & 0.49 & 0.50 & 2.02 & Putative copper-dependent oxidase \\
\hline $\mathrm{Mx} 6412$ & MXAN2146 & & & 0.32 & 0.27 & 3.14 & 0.57 & 0.80 & 1.75 & Putative calmodulin-binding protein \\
\hline Mx_1785 & MXAN1468 & & & 0.37 & 0.18 & 2.71 & 0.57 & 0.41 & 1.75 & Predicted protein \\
\hline $\mathrm{Mx} 4431$ & MXAN5074 & & & 0.37 & 0.02 & 2.68 & 0.43 & 0.30 & 2.32 & SpoVG, septation in cell division \\
\hline$M \times 4932$ & MXAN0929 & & & 0.37 & 0.30 & 2.67 & 0.49 & 0.06 & 2.03 & Hypothetical protein \\
\hline Mx_3048 & MXAN3592 & & & 0.39 & 0.04 & 2.59 & 0.39 & 0.24 & 2.56 & Ribosomal protein L35 \\
\hline$M x \_3284$ & MXAN2945 & & & 0.41 & 0.29 & 2.47 & 0.54 & 0.36 & 1.85 & Hypothetical protein \\
\hline $\mathrm{Mx} 6720$ & MXAN7433 & & & 0.42 & 0.16 & 2.40 & 0.44 & 0.26 & 2.28 & Transposase \\
\hline Mx_3811 & MXAN4788 & & & 0.43 & 0.14 & 2.34 & 0.66 & 0.28 & 1.52 & PstS \\
\hline$M \times 3042$ & MXAN2621 & & & 0.43 & 0.39 & 2.32 & 0.64 & 0.36 & 1.55 & Putative histidine kinase \\
\hline Mx_1227 & MXAN7401 & & & 0.44 & 0.21 & 2.27 & 0.48 & 0.32 & 2.09 & Vegetative protein \\
\hline Mx 5865 & MXAN6860 & & & 0.45 & 0.24 & 2.23 & 0.61 & 0.35 & 1.64 & Similar to ExbD and TolR, adventurous gliding motility proteins \\
\hline $\mathrm{M} \times 923$ & MXAN6867 & & & 0.45 & 0.25 & 2.23 & 0.66 & 0.09 & 1.51 & Peptidase \\
\hline hpkA & MXAN7512 & & & 0.46 & 0.10 & 2.19 & 0.54 & 0.08 & 1.85 & HpkA, histidine kinase \\
\hline Mx_553 & MXAN0737 & & & 0.46 & 0.17 & 2.19 & 0.57 & 0.09 & 1.75 & Polyketide synthase \\
\hline Mx_3440 & MXAN0964 & & & 0.46 & 0.18 & 2.18 & 0.36 & 0.40 & 2.74 & Calcium-binding acidic-repeat protein precursor \\
\hline Mx_2288 & MXAN0578 & & & 0.46 & 0.22 & 2.18 & 0.51 & 0.28 & 1.95 & Transposase \\
\hline Mx_5051 & MXAN4480 & & & 0.46 & 0.10 & 2.16 & 0.50 & 0.16 & 1.98 & Putative transcriptional repressor \\
\hline $\mathrm{Mx} 4270$ & MXAN1642 & & & 0.47 & 0.31 & 2.13 & 0.45 & 0.37 & 2.20 & Hypothetical protein \\
\hline Mx_4931 & MXAN0928 & & & 0.48 & 0.10 & 2.09 & 0.60 & 0.35 & 1.67 & Putative histidine kinase \\
\hline Mx_1628 & MXAN0962 & & & 0.49 & 0.31 & 2.05 & 0.49 & 0.34 & 2.04 & Calcium-binding acidic-repeat protein precursor \\
\hline Mx_2287 & MXAN0578 & & & 0.49 & 0.43 & 2.04 & 0.42 & 0.10 & 2.38 & Transposase \\
\hline \multicolumn{2}{|c|}{ Upregulated } & 48 & 60 & & & & & & & \\
\hline Mx_6280 & MXAN1724 & & & 6.31 & 10.00 & 6.31 & 203.25 & 350.86 & 203.25 & Hypothetical protein \\
\hline$M \times 6787$ & MXAN0457 & & & 4.47 & 2.90 & 4.47 & 4.59 & 4.63 & 4.59 & RedA, sigma-54 modulation domain containing protein \\
\hline Mx_5790 & MXAN1092 & & & 3.80 & 4.78 & 3.80 & 13.17 & 21.02 & 13.17 & Heat shock protein \\
\hline Mx_6923 & MXAN5892 & & & 3.42 & 2.53 & 3.42 & 1.55 & 0.41 & 1.55 & Hypothetical protein \\
\hline $\mathrm{Mx} 3763$ & MXAN2230 & & & 3.40 & 4.20 & 3.40 & 21.79 & 35.34 & 21.79 & LuxR-like, DNA binding regulator \\
\hline Mx_598 & MXAN3134 & & & 3.13 & 4.01 & 3.13 & 1.79 & 1.01 & 1.79 & Hypothetical protein \\
\hline Mx_2739 & MXAN5485 & & & 2.97 & 4.15 & 2.97 & 11.80 & 18.68 & 11.80 & Hypothetical protein \\
\hline Mx_2041 & MXAN6679 & & & 2.50 & 2.69 & 2.50 & 2.41 & 1.93 & 2.41 & Rhs family protein, cell wall associated protein \\
\hline $\mathrm{Mx} 5329$ & MXAN1578 & & & 2.46 & 3.10 & 2.46 & 9.07 & 14.31 & 9.07 & Beta-lactamase-like \\
\hline $\mathrm{Mx} \_3028$ & MXAN6573 & & & 2.43 & 2.72 & 2.43 & 2.05 & 2.47 & 2.05 & Hypothetical protein \\
\hline Mx_100 & MXAN4222 & & & 2.41 & 2.04 & 2.41 & 1.70 & 1.17 & 1.70 & Hypothetical protein \\
\hline Mx_3373.3 & MXAN6082 & & & 2.41 & 2.31 & 2.41 & 5.59 & 7.65 & 5.59 & Hypothetical protein \\
\hline $\mathrm{Mx} 6923$ & MXAN5892 & & & 2.24 & 1.62 & 2.24 & 1.86 & 1.41 & 1.86 & Hypothetical protein \\
\hline Mx_3770 & MXAN6225 & & & 2.14 & 1.58 & 2.14 & 2.45 & 1.61 & 2.45 & Hypothetical protein \\
\hline Mx_6858 & MXAN4859 & & & 2.03 & 1.25 & 2.03 & 4.01 & 4.53 & 4.01 & Fatty acid desaturase \\
\hline$M \times 2367$ & MXAN7299 & & & 2.02 & 2.01 & 2.02 & 2.65 & 3.45 & 2.65 & Fatty acid desaturase \\
\hline
\end{tabular}

Fig. 3. Genes showing at least twofold changes in expression by DNA microarray. Comparative expression in $\Delta p h o P 4$ strain VP963 with reference to the wild-type strain DK1622 is represented graphically as heat maps (blue is down-regulated in VP963, and yellow is upregulated in VP963) and as non-log-transformed expression ratios of VP963 to DK1622. Samples from three independent biological replicates were analysed, and the data within each experiment were normalized to the wildtype/mutant expression ratio at $0 \mathrm{~h}$, which is set at a ratio of 1, since PhoP4 does not appear to be active during vegetative growth (=0 h). All microarrays have been submitted to GEO (platform, GPL2848; series, GSE3795; samples, GSM87287GSM87295). Abbreviations: Av., average; SD, standard deviation. Fold changes are shown in italic type. Notes: Mx number designations are microarray spot assignments based on those of the M1 genome (Jakobsen et al., 2004; R. D. Welch, personal communication), while MXAN number designations and annotations are based on the $M$. xanthus sequence completed by The Institute for Genomic Research and the Monsanto Company.

activities, the phoP4 mutants, like other M. xanthus pho mutants, caused no defects in the vegetative phosphatase activities (data not shown).

\section{PhoP4 mutants are defective for sporulation, but not for fruiting-body formation}

Because phoP4 mutations impaired the activities of phosphatases that are active only during development, the phoP4 mutant strains were assessed for developmental phenotypes. We first tested whether the phoP4 mutants were defective for organic nutrient sensing, because earlier work has shown that $\triangle p h o R 2-p h o P 2$ cells, unlike the wild-type, fruit and sporulate when grown on media containing concentrations of $0 \cdot 2 \%$ casitone or less (Moraleda-Muñoz et al., 2003). In the case of the phoP4 mutants, this defect was not observed when either phoP4::pDV525 or $\triangle$ phoP4 cells were spotted onto media containing $0 \cdot 1,0 \cdot 2,0 \cdot 5$ or $1 \%$ casitone (data not shown). However, when sporulation was assessed on TPM starvation media, the data revealed that phoP4 mutants produced viable spore levels nearly two orders of magnitude lower than those of wild-type cells (Table 3).

\section{PhoP4 physically interacts with PhoR2}

As part of a large-scale screen of interactions among $M$. xanthus TCSs, a strong interaction was observed between the 


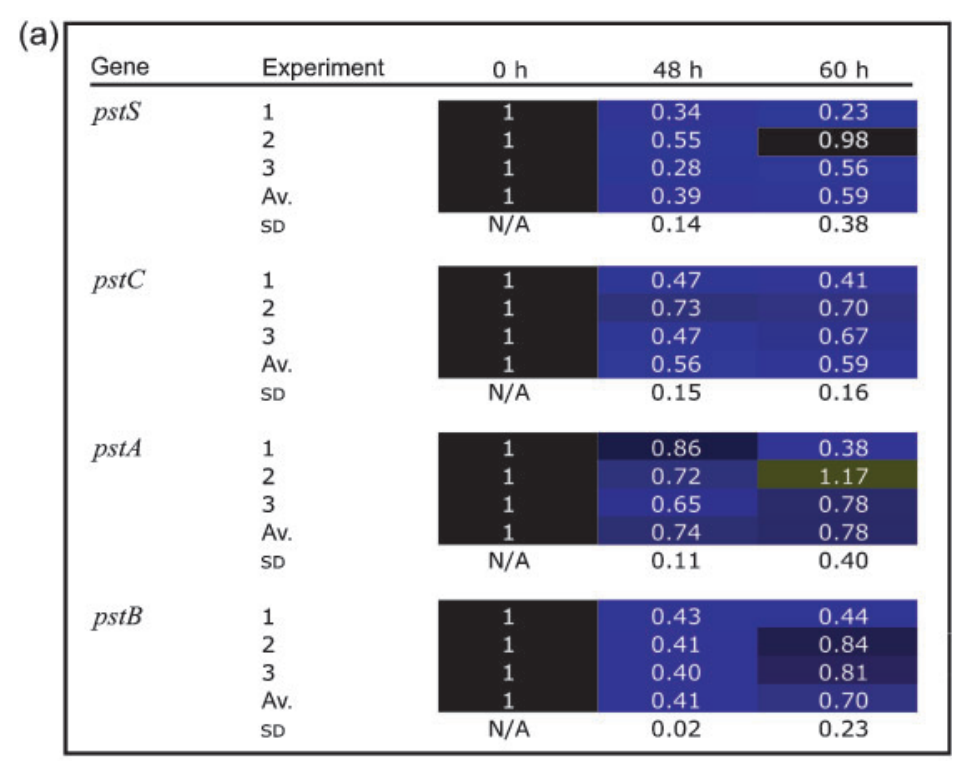

(b)

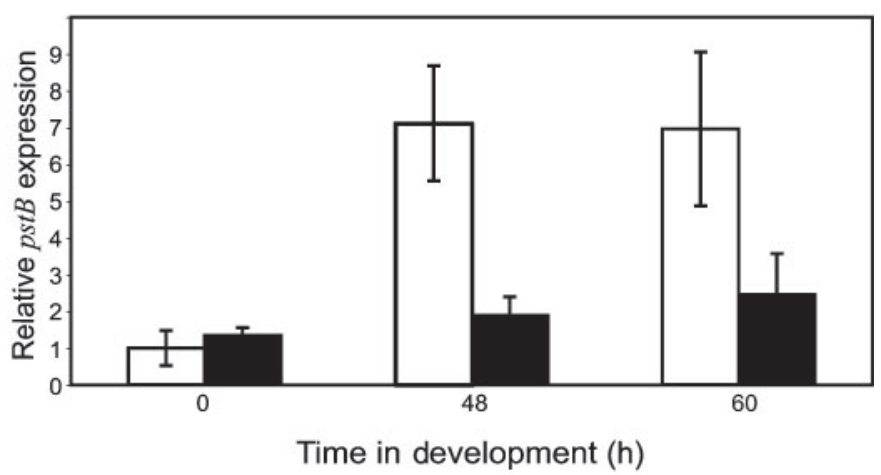

Fig. 4. PhoP4 regulation of the predicted downstream pstSCAB-phoU operon. All data were compiled from three independent biological replicates sampled as described in Methods. (a) Microarray analysis of pstS, pstC, pst $A$ and pst $B$ expression at 0,48 and $60 \mathrm{~h}$ into development. Microarray analyses were performed and submitted to GEO as described for Fig. 3. (b) QPCR analysis of pstB expression in wild-type (white bars) and $\triangle p h o P 4$ (black bars) cells at 0,48 and $60 \mathrm{~h}$ into development. Data were normalized to the wild-type level of expression at $0 \mathrm{~h}$. Parallel experiments for other genes in the predicted pstSCABphoU operon, namely pstS and phoU, showed very similar expression profiles (data not shown). orphan response regulator $\mathrm{PhoP} 4$ and the histidine kinase PhoR2. Three Pho-type systems have been previously described in M. xanthus (Pho1, Pho2 and Pho3), each composed of a histidine kinase and a response regulator. The response regulators are very similar to each other, and to PhoP4 (Fig. 1). Cross-communication among different Pho regulon TCSs is known in other systems (Amemura et al., 1990), and therefore interactions among the $M$. xanthus Pho TCS proteins were systematically tested using the yeast two-hybrid system.

The results showed strong and specific interactions among some of the Pho protein fusions (Table 4). Strong reciprocal interactions were observed between PhoP1 and PhoR1, as would be expected of true TCS partners, while PhoR1 interacted with itself, suggesting homodimerization. An expected interaction between the protein fusions of the putative TCS partners PhoP2 and PhoR2 was also observed, though this interaction was not reciprocal. The PhoP2 receiver domain was apparently able to interact with itself, which is known to be the case for some response regulators (Lewis et al., 2002; Muller-Dieckmann et al., 1999). No interactions were seen for the Pho3 proteins, except that PhoR3 interacted strongly with PhoP2, which is not surprising given the high similarity of the Pho2 and Pho3 systems (Moraleda-Muñoz et al., 2003). Some other predicted interactions for these two systems were also not observed (or were not reciprocal), suggesting a significant rate of false negatives in the assay results.

Most interestingly, the PhoP4 fusion proteins, regardless of whether assayed as bait or prey, interacted strongly with both PhoR2 fusions. Such reciprocal interactions provide solid evidence of a PhoP4-PhoR2 interactional relationship. While PhoP4 also interacted with both the PhoP1 and PhoP2 fusions, these interactions were not reciprocal, and their significance must therefore be considered more warily; this is also true for the non-reciprocal PhoP2-PhoR3 and PhoP2-PhoR2 interactions.

\section{DISCUSSION}

Studying signalling pathways is crucial for understanding how cells sense and respond to environmental stimuli. The soil bacterium M. xanthus possesses 255 components predicted to participate in pathways involved in signal reception and transduction (D. E. Whitworth, unpublished data). At least four of these pathways regulate Pho regulon 
(a) Acid phosphatase activity

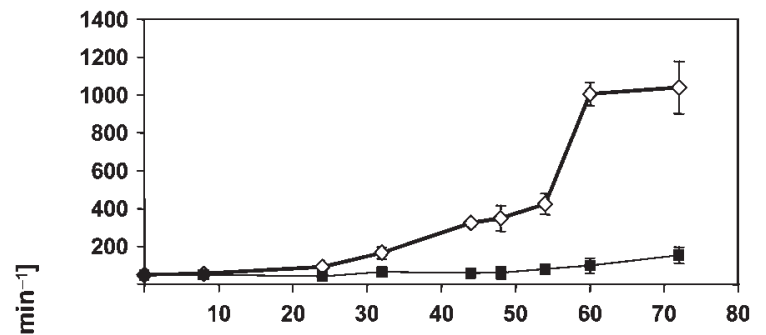

(b) Neutral phosphatase activity

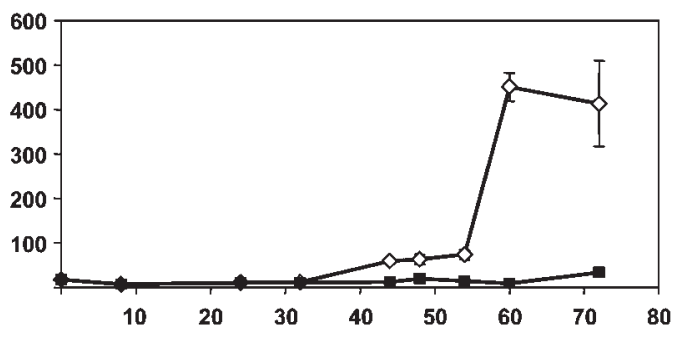

(c) Alkaline phosphatase activity

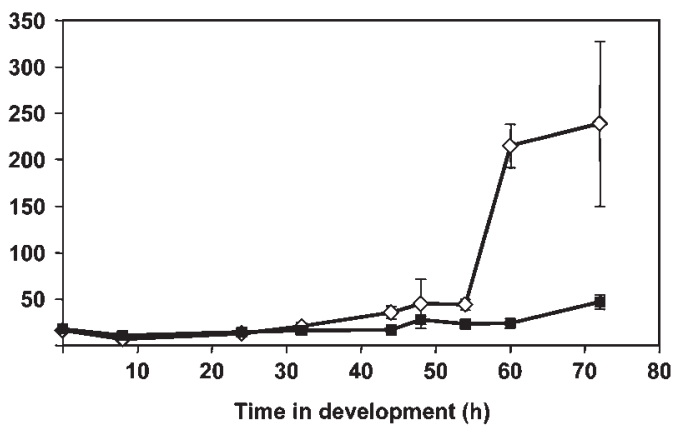

Fig. 5. Effect of phoP4 mutations on regulation of development-specific phosphatase activities. (a) Acid, (b) neutral, and (c) alkaline phosphatase activities. Wild-type $(\diamond)$ and phoP4 (ם) cells were induced to undergo development on CF starvation agar. Threefold replicate samples were harvested at various time-points between 0 and $72 \mathrm{~h}$ into development, and phosphatase activities at $\mathrm{pH} 5.2,7.2$ and 8.5 were separately monitored by the production of $p$-nitrophenol (pNP). The phosphatase activity curves for VP963 ( $\Delta$ phoP4) and VP965 (phoP4::pDV525) cells were nearly identical, and only the VP965 results are shown to maintain figure clarity. components in M. xanthus, possibly because having multiple regulatory systems allows $M$. xanthus to fine-tune more precisely the relevant responses associated with the maintenance of internal phosphate pools.

The data in this study showed that the function of one Pho TCS, represented here by $\mathrm{PhoP} 4$, is tied closely to processes linked to phosphate regulation. Not only did phoP4 mutations decrease spore viability by nearly two orders of magnitude (Table 3), perhaps by disrupting the developmental response to overall phosphate starvation, but they also impaired the magnesium-independent phosphatase activities that are associated with myxospores (Fig. 5). Furthermore, mutating phoP4 also decreased expression of the predicted downstream pstSCAB-phoU operon (Fig. 4), which is homologous to systems responsible for inorganic phosphate assimilation (Wanner, 1996). Interestingly, the PhoP4-dependent Pst system appeared to be active very late in development (at $60 \mathrm{~h}$ ), when most M. xanthus cells either have lysed or are developing into myxospores (Julien et al., 2000). Possibly, the scavenging of inorganic phosphate by the Pst system at this stage, combined with the co-occurring high phosphatase activities, may be important for spore formation; this possibility is further strengthened by the observed defect in spore viability for the phoP4 mutants. Moraleda-Muñoz et al. (2003) have reported that a $\Delta p h o R 2-$ phoP2 $\Delta$ phoR3-phoP3 double mutant, while having no defect in overall spore viability, nevertheless does produce a fraction of myxospores (5-10\%) that do not reshape completely: they are ellipsoid rather than being roughly round like wild-type myxospores. Similar high-resolution microscopic imaging of the few phoP4 myxospores produced may reveal whether structural defects are caused by the pho4 mutations. Any defects found will likely be more severe than the simple misshaping of a fraction of the phoP4 myxospores formed (given the observed deficit in spore viability), and may include deformities in the spore coat, intermediate coat or cortex. Indeed, visual inspection of phoP4 myxospores by phase-contrast microscopy revealed no discernible differences compared to wild-type myxospores in terms of phase brightness, shape or size.

Beyond examining the various phenotypes caused by phoP4 mutants, which are quantitatively the strongest among the $M$. xanthus pho mutants so far reported, the question

Table 3. Spore viability assay

Spores were harvested at $72 \mathrm{~h}$ after development was initiated by spotting $5 \times 10^{8}$ cells onto TPM starvation agar, as described in Methods. Values shown in the 'No. of viable spores' column represent the means of three independent experiments.

\begin{tabular}{|lcc|}
\hline Strain & No. of viable spores & As a percentage of wild-type (mean \pm range) \\
\hline Wild-type & $8 \cdot 90 \times 10^{6}$ & $100 \cdot 0 \pm 6 \cdot 7$ \\
phoP4::pDV525 & $3 \cdot 36 \times 10^{5}$ & $3 \cdot 8 \pm 1 \cdot 1$ \\
SphoP4 & $1 \cdot 34 \times 10^{5}$ & $1 \cdot 5 \pm 0 \cdot 3$ \\
\hline
\end{tabular}


Table 4. Yeast two-hybrid interactions

Pairwise yeast two-hybrid interactions between bait and prey protein fusions were conducted as indicated in Methods. The strength of interaction, based on the intensity of the reporter indigo colour, was scored on a scale of $1-3$, with a 3 assigned to the greatest colour intensity and hence the strongest interaction. No interaction is signified by -. See Methods for corresponding $\beta$-galactosidase specific activity values. Interactions involving PhoP4 or PhoR2 are highlighted in bold type. The interaction between RNM and QN was used as a positive control against which experimental interactions were standardized. $\mathrm{AD}$, activation domain.

\begin{tabular}{|c|c|c|c|c|c|c|c|c|}
\hline \multirow[t]{2}{*}{ LexA-bait fusion } & \multicolumn{8}{|c|}{ AD-prey fusion } \\
\hline & RNM & PhoP1 & PhoR1 & PhoP2 & PhoR2 & PhoP3 & PhoR3 & PhoP4 \\
\hline QN & 3 & - & - & - & - & - & - & - \\
\hline PhoP1 & - & - & 3 & - & - & - & - & 2 \\
\hline PhoR1 & - & 2 & 3 & - & - & - & - & - \\
\hline PhoP2 & - & - & - & 3 & - & - & - & 2 \\
\hline PhoR2 & - & - & - & 3 & - & - & - & 3 \\
\hline PhoP3 & - & - & - & - & - & - & - & - \\
\hline PhoR3 & - & - & - & 3 & - & - & - & - \\
\hline PhoP4 & - & - & - & - & 2 & - & - & - \\
\hline
\end{tabular}

regarding the identity of the histidine kinase partner(s) to PhoP4 has yet to be fully answered. We noted that, whereas the mutants of each M. xanthus Pho TCS displayed different complements of phenotypes (Carrero-Lerida et al., 2005; Martinez-Canamero et al., 2003; Moraleda-Muñoz et al., 2003), they all nevertheless affected certain subsets of the as-yet unidentified, developmentally active phosphatases (Weinberg \& Zusman, 1990). This suggested to us that the four Pho TCSs may be functionally interlinked, rather than acting entirely separately from one another. Intriguingly, as reported above, a yeast two-hybrid analysis for interactions among the seven known Pho TCS components showed strong, reciprocal interactions between the receiver and H-box domains of PhoP4 and PhoR2, respectively (Table 4). It is possible that inputs from signals that activate the Pho2 and Pho4 systems, if received simultaneously, may lead to cross-communication between them, and amplify the expression of genes under their common control, e.g. those connected with the acid phosphatase activities.

However, besides interacting with PhoR2, we hypothesize that PhoP4 must be partnered with at least one other histidine kinase to regulate sporulation, and with the developmental alkaline and neutral phosphatase activities, since these processes are not affected in the phoR2 mutant. The recently completed genome of $M$. xanthus may allow for a systematic approach towards identifying this histidine kinase partner of PhoP4. Since PhoP4 is a homologue of E. coli $\mathrm{PhoB}$, it is predicted that its histidine kinase partner would resemble E. coli PhoR, the PhoB partner. The $M$. xanthus genome has been parsed for TCS components, and they have been classified based on domain architecture, gene order and deduced sequence phylogenies (D. E. Whitworth $\&$ D. A. Hodgson, unpublished results). These analyses have revealed a grouping of 13 histidine kinases, termed class II histidine kinases, which share these features with E. coli PhoR. In the next step, yeast two-hybrid analyses similar to the ones described in this study will be used to assess whether any of these components interact with PhoP4. Coupled with expression studies to evaluate the physiological relevance of any interactions, and phenotypic analyses comparing phoP4 mutants to mutants in genes of candidate partners, these future experiments will all help decipher the complexity of the M. xanthus phosphate regulatory circuitry.

\section{ACKNOWLEDGEMENTS}

We are grateful to the Monsanto Company and The Institute for Genomic Research for access to the M. xanthus genome sequence prior to publication. This work was supported, in part, by a Floyd and Mary Schwall Medical Research Fellowship and Public Health Service grant T32GM0737 to V.D.P, and by Public Health Service grant GM54592 to M.S. D.E.W. was supported by grant P16665 from the Biotechnology and Biological Sciences Research Council (BBSRC), UK.

\section{REFERENCES}

Amemura, M., Makino, K., Shinagawa, H. \& Nakata, A. (1990). Cross talk to the phosphate regulon of Escherichia coli by PhoM protein: PhoM is a histidine protein kinase and catalyzes phosphorylation of PhoB and PhoM-open reading frame 2. J Bacteriol 172, 6300-6307.

Antelmann, H., Scharf, C. \& Hecker, M. (2000). Phosphate starvationinducible proteins of Bacillus subtilis: proteomics and transcriptional analysis. J Bacteriol 182, 4478-4490.

Blattner, F. R. \& Plunkett, G., 3rd, Bloch, C. A. \& 14 other authors (1997). The complete genome sequence of Escherichia coli K-12. Science 277, 1453-1474.

Browning, D. F., Whitworth, D. E. \& Hodgson, D. A. (2003). Lightinduced carotenogenesis in Myxococcus xanthus: functional characterization of the ECF sigma factor CarQ and antisigma factor CarR. Mol Microbiol 48, 237-251.

Carrero-Lerida, J., Moraleda-Muñoz, A., Garcia-Hernandez, R., Perez, J. \& Muñoz-Dorado, J. (2005). PhoR1-PhoP1, a third twocomponent system of the family PhoRP from Myxococcus xanthus: role in development. J Bacteriol 187, 4976-4983. 
Chou, P. Y. \& Fasman, G. D. (1978). Prediction of the secondary structure of proteins from their amino acid sequence. Adv Enzymol Relat Areas Mol Biol 47, 45-148.

Diodati, M. E., Ossa, F., Caberoy, N. B., Jose, I. R., Hiraiwa, W., Igo, M. M., Singer, M. \& Garza, A. G. (2006). Nla18, a key regulatory protein required for normal growth and development of Myxococcus xanthus. J Bacteriol 188, 1733-1743.

Gyuris, J., Golemis, E., Chertkov, H. \& Brent, R. (1993). Cdi1, a human G1 and S phase protein phosphatase that associates with Cdk2. Cell 75, 791-803.

Hagen, D. C., Bretscher, A. P. \& Kaiser, D. (1978). Synergism between morphogenetic mutants of Myxococcus xanthus. Dev Biol 64, 284-296.

Harris, B. Z., Kaiser, D. \& Singer, M. (1998). The guanosine nucleotide (p)ppGpp initiates development and A-factor production in Myxococcus xanthus. Genes Dev 12, 1022-1035.

Higgs, P. I., Cho, K., Whitworth, D. E., Evans, L. S. \& Zusman, D. R. (2005). Four unusual two-component signal transduction homologs, RedC to RedF, are necessary for timely development in Myxococcus xanthus. J Bacteriol 187, 8191-8195.

Hirani, T. A., Suzuki, I., Murata, N., Hayashi, H. \& Eaton-Rye, J. J. (2001). Characterization of a two-component signal transduction system involved in the induction of alkaline phosphatase under phosphate-limiting conditions in Synechocystis sp. PCC 6803. Plant Mol Biol 45, 133-144.

Hodgkin, J. \& Kaiser, D. (1977). Cell-to-cell stimulation of movement in nonmotile mutants of Myxococcus. Proc Natl Acad Sci U S A 74, 2938-2942.

Hulett, F. M., Lee, J., Shi, L., Sun, G., Chesnut, R., Sharkova, E., Duggan, M. F. \& Kapp, N. (1994). Sequential action of twocomponent genetic switches regulates the PHO regulon in Bacillus subtilis. J Bacteriol 176, 1348-1358.

Jakobsen, J. S., Jelsbak, L., Welch, R. D., Cummings, C., Goldman, B., Stark, E., Slater, S. \& Kaiser, D. (2004). $\sigma^{54}$ enhancer binding proteins and Myxococcus xanthus fruiting body development. J Bacteriol 186, 4361-4368.

Julien, B., Kaiser, A. D. \& Garza, A. (2000). Spatial control of cell differentiation in Myxococcus xanthus. Proc Natl Acad Sci U S A 97, 9098-9103.

Kaiser, D. (1979). Social gliding is correlated with the presence of pili in Myxococcus xanthus. Proc Natl Acad Sci U S A 76, 5952-5956.

Kaiser, D. (2000). Cell-interactive sensing of the environment. In Prokaryotic Development, pp. 263-275. Edited by Y. V. Brun and L. J. Shimkets. Washington, DC: American Society for Microbiology.

Karp, P. D., Riley, M., Saier, M., Paulsen, I. T., Collado-Vides, J., Paley, S. M., Pellegrini-Toole, A., Bonavides, C. \& Gama-Castro, S. (2002). The EcoCyc database. Nucleic Acids Res 30, 56-58.

Kashefi, K. \& Hartzell, P. L. (1995). Genetic suppression and phenotypic masking of a Myxococcus xanthus frzF $F^{-}$defect. Mol Microbiol 15, 483-494.

Kenney, L. J. (2002). Structure/function relationships in OmpR and other winged-helix transcription factors. Curr Opin Microbiol 5, 135-141.

Kroos, L., Kuspa, A. \& Kaiser, D. (1986). A global analysis of developmentally regulated genes in Myxococcus xanthus. Dev Biol 117, 252-266.

Lewis, R. J., Scott, D. J., Brannigan, J. A., Ladds, J. C., Cervin, M. A., Spiegelman, G. B., Hoggett, J. G., Barak, I. \& Wilkinson, A. J. (2002). Dimer formation and transcription activation in the sporulation response regulator Spo0A. J Mol Biol 316, 235-245.
Liu, W. \& Hulett, F. M. (1998). Comparison of PhoP binding to the tuaA promoter with PhoP binding to other Pho-regulon promoters establishes a Bacillus subtilis Pho core binding site. Microbiology 144, 1443-1450.

Makino, K., Shinagawa, H., Amemura, M., Kawamoto, T., Yamada, M. \& Nakata, A. (1989). Signal transduction in the phosphate regulon of Escherichia coli involves phosphotransfer between PhoR and PhoB proteins. J Mol Biol 210, 551-559.

Makino, K., Amemura, M., Kawamoto, T., Kimura, S., Shinagawa, H., Nakata, A. \& Suzuki, M. (1996). DNA binding of PhoB and its interaction with RNA polymerase. J Mol Biol 259, 15-26.

Manoil, C. \& Kaiser, D. (1980). Guanosine pentaphosphate and guanosine tetraphosphate accumulation and induction of Myxococcus xanthus fruiting body development. J Bacteriol 141, 305-315.

Martinez-Canamero, M., Ortiz-Codorniu, C., Extremera, A. L., Muñoz-Dorado, J. \& Arias, J. M. (2003). phoR1, a gene encoding a new histidine protein kinase Myxococcus xanthus. Antonie Van Leeuwenhoek 83, 361-368.

Moraleda-Muñoz, A., Carrero-Lérida, J., Pérez, J. \& Muñoz-Dorado, J. (2003). Role of two novel two-component regulatory systems in development and phosphatase expression in Myxococcus xanthus. J Bacteriol 185, 1376-1383.

Muller-Dieckmann, H. J., Grantz, A. A. \& Kim, S. H. (1999). The structure of the signal receiver domain of the Arabidopsis thaliana ethylene receptor ETR1. Structure 7, 1547-1556.

Okamura, H., Hanaoka, S., Nagadoi, A., Makino, K. \& Nishimura, Y. (2000). Structural comparison of the PhoB and OmpR DNAbinding/transactivation domains and the arrangement of PhoB molecules on the phosphate box. J Mol Biol 295, 1225-1236.

Parkinson, J. S. \& Kofoid, E. C. (1992). Communication modules in bacterial signaling proteins. Annu Rev Genet 26, 71-112.

Pham, V. D., Shebelut, C. W., Zumstein, E. J. \& Singer, M. (2005). $\mathrm{BrgE}$ is a regulator of Myxococcus xanthus development. Mol Microbiol 57, 762-773.

Romeo, J. M., Esmon, B. \& Zusman, D. R. (1986). Nucleotide sequence of the myxobacterial hemagglutinin gene contains four homologous domains. Proc Natl Acad Sci U S A 83, 6332-6336.

Saldanha, A. J. (2004). Java Treeview - extensible visualization of microarray data. Bioinformatics 20, 3246-3248.

Sambrook, J., Fritsch, E. F. \& Maniatis, T. (1989). Molecular Cloning: a Laboratory Manual, 2nd edn. Cold Spring Harbor, NY: Cold Spring Harbor Laboratory.

Shimkets, L. J. (1999). Intercellular signaling during fruitingbody development of Myxococcus xanthus. Annu Rev Microbiol 53, 525-549.

Spratt, B. G., Hedge, P. J., te Heesen, S., Edelman, A. \& BroomeSmith, J. K. (1986). Kanamycin-resistant vectors that are analogues of plasmids pUC8, pUC9, pEMBL8 and pEMBL9. Gene 41, 337-342.

Stock, A. M., Robinson, V. L. \& Goudreau, P. N. (2000). Twocomponent signal transduction. Annu Rev Biochem 69, 183-215.

Thompson, J. D., Higgins, D. G. \& Gibson, T. J. (1994). CLUSTAL W: improving the sensitivity of progressive multiple sequence alignment through sequence weighting, position-specific gap penalties and weight matrix choice. Nucleic Acids Res 22, 4673-4680.

Tusher, V. G., Tibshirani, R. \& Chu, G. (2001). Significance analysis of microarrays applied to the ionizing radiation response. Proc Natl Acad Sci U S A 98, 5116-5121.

Ueki, T., Inouye, S. \& Inouye, M. (1996). Positive-negative KG cassettes for construction of multi-gene deletions using a single drug marker. Gene 183, 153-157. 
Wanner, B. L. (1996). Phosphorus assimilation and control of the phosphate regulon. In Escherichia coli and Salmonella: Cellular and Molecular Biology, pp. 1357-1381. Edited by F. C. Neidhardt. Washington, DC: American Society for Microbiology.

Ward, M. J. \& Zusman, D. R. (2000). Developmental aggregation and fruiting body formation in the gliding bacterium Myxococcus xanthus. In Prokaryotic Development, pp. 243-262. Edited by Y. V. Brun and L. J. Shimkets. Washington, DC: American Society for Microbiology.
Weinberg, R. A. \& Zusman, D. R. (1990). Alkaline, acid, and neutral phosphatase activities are induced during development in Myxococcus xanthus. J Bacteriol 172, 2294-2302.

Whitworth, D. E. \& Hodgson, D. A. (2001). Light-induced carotenogenesis in Myxococcus xanthus: evidence that CarS acts as an anti-repressor of CarA. Mol Microbiol 42, 809-819.

Yanisch-Perron, C., Vieira, J. \& Messing, J. (1985). Improved M13 phage cloning vectors and host strains: nucleotide sequences of the M13mp18 and pUC19 vectors. Gene 33, 103-119. 